\title{
Erratum: Human embryonic stem cell-derived cardiomyocytes restore function in infarcted hearts of non-human primates
}

Yen-Wen Liu, Billy Chen, Xiulan Yang, James A Fugate, Faith A Kalucki, Akiko Futakuchi-Tsuchida, Larry Couture, Keith W Vogel, Clifford A Astley, Audrey Baldessari, Jason Ogle, Creighton W Don, Zachary L Steinberg, Stephen P Seslar, Stephanie A Tuck, Hiroshi Tsuchida, Anna V Naumova, Sarah K Dupras, Milly S Lyu, James Lee, Dale W Hailey, Hans Reinecke, Lil Pabon, Benjamin H Fryer, W Robb MacLellan, R Scott Thies \& Charles E Murry Nat. Biotechnol. 36, 597-605 (2018); published online 2 July 2018; corrected after print 18 July 2018

In the version of this article initially published, NIH grant P51 OD010425 was omitted. The error has been corrected in the HTML and PDF versions of the article.

\section{Erratum: Comprehensive multi-center assessment of small RNA-seq methods for quantitative miRNA profiling}

Maria D Giraldez, Ryan M Spengler, Alton Etheridge, Paula M Godoy, Andrea J Barczak, Srimeenakshi Srinivasan, Peter L De Hoff, Kahraman Tanriverdi, Amanda Courtright, Shulin Lu, Joseph Khoory, Renee Rubio, David Baxter, Tom A P Driedonks, Henk P J Buermans, Esther N M Nolte-'t Hoen, Hui Jiang, Kai Wang, Ionita Ghiran, Yaoyu E Wang, Kendall Van Keuren-Jensen, Jane E Freedman, Prescott G Woodruff, Louise C Laurent, David J Erle, David J Galas \& Muneesh Tewari Nat. Biotechnol. doi:10.1038/nbt.4183; corrected online 31 July 2018

In the version of this article initially published online, the text "Beth Israel Deaconess Medical Center/Dana Farber Cancer Institute (BIDMC/ DFCI)" was inserted into the last sentence in the right-hand column of p.10, beginning "It is worth noting...." . In addition, on p.2, the acronym for The Cancer Genome Atlas was given as TGCA, rather than TCGA; and on p. 3, UUTR should have been defined, as University of Utrecht, the

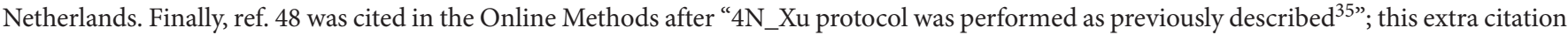
has been deleted. The errors have been corrected for the print, PDF and HTML versions of this article.

\section{Erratum: Repair of double-strand breaks induced by CRISPR-Cas9 leads to large deletions and complex rearrangements}

Michael Kosicki, Kärt Tomberg \& Allan Bradley

Nat. Biotechnol. doi:10.1038/nbt.4192; corrected online 31 July 2018

In the version of this article initially published online, four figure citations were incorrect on p.2: left-hand column, after "complex rearrangements," "Supplementary Fig. 2a,b" should have been "Fig. 2a,b"; right-hand column, in three places, the citation for "Supplementary Fig. 3..." should have been for "Supplementary Fig. 2." The errors have been corrected for the print, PDF and HTML versions of this article.

\section{Erratum: Strength in numbers from integrated single-cell neuroscience}

Rahul Satija

Nat. Biotechnol. 36, 41-42 (2018); published online 10 January 2018; corrected after print 7 August 2018

In the version of this article initially published, the sentence fragment "The scNuc-seq approach is complementary to the recently published massively parallel” preceding "The sNuc-seq approach is complementary to the recently published DroNc-seq method" should have been deleted. The error has been corrected in the HTML and PDF versions of the article. 